\title{
miR-106b Promotes Metastasis of Early Gastric Cancer by Targeting ALEX1 in Vitro and in Vivo
}

\author{
Zhenglun Zhu ${ }^{\mathrm{a}}$ Qiumeng Yang ${ }^{\mathrm{a}}$ Benyan Zhang ${ }^{\mathrm{b}}$ Wei Wu ${ }^{\mathrm{c}}$ Fei Yuan ${ }^{\mathrm{b}}$ \\ Zhenggang Zhu ${ }^{a}$ \\ aDepartment of Surgery, Ruijin Hospital, Shanghai Jiao Tong University School of Medicine, Shanghai, \\ China, 'Department of Pathology, Ruijin Hospital, Shanghai Jiao Tong University School of Medicine, \\ Shanghai, China, 'Department of Gastroenterology, Ruijin Hospital, Shanghai Jiao Tong University \\ School of Medicine, Shanghai, China
}

\section{Key Words}

miR-106b • ALEX1 • EGC • Metastasis • JAK1/STAT3 signaling pathway • GLPG0643

\begin{abstract}
Background/Aims: Aberrant expression of miR-106b is a specific symptom of many solid carcinomas. Overexpression of miR-106b has been observed in gastric cancer. The effect of miR-106b on gastric cancer has been investigated in different cell culture models. However, the effect of miR-106b on metastasis of early gastric cancer (EGC) remains unknown. Methods: In the study, qRT-PCR, FISH, western blot, luciferase reporter assay, migration and invasion assays, flow cytometry and TUNEL staining were used to investigate the effect of miR-106b on metastasis of EGC. Results: To explore the function of miR-106b in EGC, we investigated the downstream signaling of miR-106b and found that ALEX1 was a direct target of miR-106 in gastric cancer cells. Up-regulation of ALEX1 effectively rescued the cell apoptosis induced by miR-106b inhibitor and promoted the expression levels of phosphorylation of JAK1 and STAT3. Moreover, overexpression of JAK1 reduced the cell apoptosis induced by miR-106b inhibitor and decreased the expression levels of the apoptotic proteins in gastric cancer cells. Furthermore, down-regulation of miR-106b promoted apoptosis of gastric cancer cells via inhibiting JAK1/STAT3 signaling pathway in vitro and in vivo. In addition, GLPG0643, a JAK1 inhibitor, enhanced the inhibitory effect of miR-106b inhibitor on gastric cancer growth in vivo. Conclusion: These findings provided a potential therapeutic manner for the treatment of metastasis of EGC in clinic.
\end{abstract}

Z. Zhu and Q. Yang contributed equally to this work.

$\begin{array}{ll}\text { Zhenggang Zhu } & \text { Department of Pathology, Ruijin Hospital, Shanghai Jiao Tong University } \\ \text { and Fei Yuan } & \text { School of Medicine, Shanghai (China) } \\ & \text { E-Mail zhenggang003@yeah.net }\end{array}$




\section{Cellular Physiology Cell Physiol Biochem 2019;52:606-616 \\ \begin{tabular}{ll|l} 
and Biochemistry & $\begin{array}{l}\text { DOl: 10.33594/000000043 } \\
\text { Published online: 26 March 2019 }\end{array}$ & $\begin{array}{l}\text { O } 2019 \text { The Author(s). Published by } \\
\text { Cell Physiol Biochem Press GmbH\&Co. KG }\end{array}$ \\
\cline { 2 - 3 } &
\end{tabular} \\ Zhu et al.: Effect of miR-106b on Metastasis of Early Gastric Cancer}

\section{Introduction}

Gastric cancer is one of the most common malignancies in the world. The incidence rate of gastric cancer has been gradually increasing in recent years [1,2]. The diagnosis and treatment of early gastric cancer (EGC) are faced with huge challenges, which have caused widespread concern in the world [3-6]. EGC is defined as the invasion of stomach limited to the mucosal layer and submucosa and it has no direct correlation with lymph node metastasis [7]. Currently, the metastasis of EGC, the main cause that affects the survival of the patients, has no effective treatment manners [8-10]. Additionally, EGC is difficult to diagnose due to the non-specific symptoms. Although the application of endoscopic submucosal dissection can effectively improve the surgical outcome, the incidence rate of lymph node metastasis is still highly occurred, which achieves 5\%-28\% in patients with EGC [11, 12]. Thus, a deeper understanding of the molecular mechanism of the invasion and metastasis may lead to the better diagnosis and treatment of EGC.

miRNAs are a class of non-coding RNAs that act on posttranscriptional translation by targeting the target protein, which affect a wide range of biological functions such as cell proliferation, differentiation and apoptosis [13-15]. Different types of miRNAs may play different roles such as oncogenes or tumor suppressor genes in the tumorgenesis and development of gastric cancer [16-18]. Previous studies have shown that miR-106b promotes the occurrence of tumor metastasis in liver cancer and colorectal cancer $[19,20]$. Moreover, the expression of miR-106b in serum can be used as a marker of high risk of EGC in a previous study [21]. Recent studies have shown that miR-106b has been involved in the regulation of invasion and migration of different tumor cell lines [22, 23]. However, the role of miR-106b in the invasion and metastasis of EGC and its detailed mechanism remain unclear. Arm protein lost in epithelial cancers, on chromosome X (ALEX) is a subgroup of armadillo family, which participates in a variety of cell processes such as cell proliferation, adhesion and apoptosis [24]. A recent study has revealed that overexpression of ALEX1 can inhibit the cell proliferation and induce the cell apoptosis, whereas depletion of ALEX1 leads to the increasing proliferation and the inhibitory apoptosis in breast cancer cells [25]. Hiroyoshi Iseki et al. found that overexpression of ALEX1 suppresses the colony formation in human colorectal carcinoma cells [24]. However, the relationship between ALEX1 and miR$106 \mathrm{~b}$ and the probably mechanism in the metastasis of EGC are urgently needed to be solved. Here, we evaluate the function of miR-106b in EGC and investigate the mechanism of miR106b mediated metastasis in vitro and in vivo. The findings will help reduce the incidence of EGC metastasis, and provide new ideas for the treatment of metastasis of EGC in clinic.

\section{Materials and Methods}

\section{Patients and Specimens}

The metastasis and non-metastasis tumor tissues were acquired from the patients with EGC. The patients with EGC underwent surgical resection at the Ruijin Hospital of Shanghai Jiaotong University. These patients did not have history of chemotherapy or radiotherapy before collecting specimens. The specimens were collected with the consent of the patients. The experiment was approved by the Ethics Committee of Shanghai Jiaotong University.

\section{Cell culture}

Human gastric cancer cell AGS was acquired from Chinese Academy of Sciences and cultured in RPMI1640 supplemented with 10\% fetal bovine serum (FBS; Hyclone, Logan,UT, USA). The cells were maintained at $37^{\circ} \mathrm{C}$ in an atmosphere of $5 \% \mathrm{CO}_{2}$. 


\section{Cellular Physiology Cell Physiol Biochem 2019;52:606-616

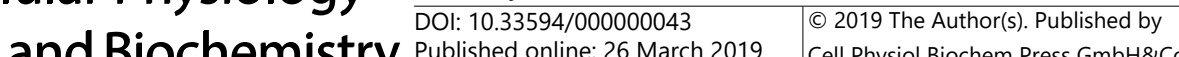 \\ Published online: 26 March 2019 Cell Physiol Biochem Press GmbH\&Co. KG \\ Zhu et al.: Effect of miR-106b on Metastasis of Early Gastric Cancer}

\section{Quantitative reverse transcription polymerase chain reaction ( $q R T-P C R$ )}

Total RNA was extracted using TRLZOL reagent (Invitrogen). Reverse transcription was performed and cDNA was prepared for amplification. The expression levels of miR-106b were quantified using a miRNAspecific TaqMan miRNA Assay Kit (Applied Biosystems). The reaction was performed with incubation at $95^{\circ} \mathrm{C}$ for $5 \mathrm{~min}, 95^{\circ} \mathrm{C}$ for $30 \mathrm{~s}, 58^{\circ} \mathrm{C}$ for $30 \mathrm{~s}$, and $72^{\circ} \mathrm{C}$ for $30 \mathrm{~s}\left(36 \mathrm{cycles}\right.$ ), and at $72^{\circ} \mathrm{C}$ for $10 \mathrm{~min}$ using the primers (F:5'-AGCCGTCAAGAGCAATAACGAA-3', R: 5'-GTGCAGGG TCCGAGGT-3'). U6 was used as a reference gene. Analysis of expression levels of miR-106b was performed in triplicate and calculated using the 2- $\Delta \Delta \mathrm{ct}$ method.

\section{Fluorescence in situ hybridization (FISH)}

Tissue was prepared and performed ISH using the miR-106b probe (5'-ATCTGCACTGTCAGCACTTTA-3' and the microRNA ISH Kit (Exiqon, Vedbaek, Denmark). The signals of IF were examined with a fluorescence microscope (Olympus).

\section{Transwell migration and invasion assay}

Cell suspension was added into the upper cup of transwell chambers (Corning Incorporated, NY, USA). The lower chamber was supplied with 10\% FBS-supplemented media. After $16 \mathrm{~h}$, non-migrating cells inside upper cup were removed by cotton swab. Cells migrating through the membrane to bottom surface of the cup were fixed in methanol, stained with $0.5 \%$ crystal violet, and subjected to microscopic analysis. Numbers of migrating cells were counted in ten randomly selected fields. Invasion assay was conducted using 24-well transwell chambers coated with $3 \mathrm{mg} / \mathrm{ml}$ matrigel (Corning Incorporated, NY). The invading cells were analyzed after $24 \mathrm{~h}$.

\section{Luciferase Reporter assays}

The promoter of ALEX1 was cloned in pGL 3.0 luciferase reporter plasmids. Cells were cotransfected with pRL-CMV renilla luciferase reporter and the pGL 3.0 luciferase reporter plasmid containing the promoters of ALEX1. The luciferase activities were measured by a dual luciferase reporter assay system (Promega).

\section{Lentivirus vector construction, transfection and infection}

HEK-293T cells were fostered in culture dish. The lentivirus vectors containing ALEX1 (PLVX-ALEX1) and JAK1 (PLVX-JAK1) were transfected into HEK-293T. Viral lysates were harvested and purified. After AGS cells were incubated in plate for $24 \mathrm{~h}$. PLVX vectors were added into the medium and infected the cells.

\section{Western blot}

For western blots, samples were separated on SDS-PAGE gels and then transferred to PVDF membranes (Millipore). After blocking with milk, membranes were processed following the ECL Western blotting protocol (GE). Bcl-2, Bax, Bim, ZEB1, SNAI2, TWIST1, E-cadherin, pJAK1, pSTAT3 and GAPDH antibodies were acquired from Cell Signaling Technology (Beverly, MA, USA). ALEX1 antibody was obtained from Abcam (Cambridge, MA).

\section{Flow cytometry}

Cell suspension was prepared and added $10 \mu \mathrm{L}$ of Annexin V, incubated in dark for 15 min, then added $5 \mu \mathrm{L}$ of 7AAD, and analyzed using the flow cytometer within 5 minutes. The results were expressed as percentage of apoptotic cells.

\section{Animals}

BALB/c nude mice were used in the experiments at the age of 4 weeks. The mice were provided from the Animal Center of the Second Military Medical University. All animal experiments were conducted in strict accordance with the guidelines for the care and use of experimental animals and were approved by the Animal Care Committee of Ruijin Hospital, Shanghai Jiaotong University. Mice bearing AGS tumor xenografts were randomly divided into five groups: Control, NC, GLPG0634, miR-106b inhibitor, miR-106b inhibitor + GLPG0634. Volumes of tumor were measured every week. 


\section{Cellular Physiology Cell Physiol Biochem 2019;52:606-616 \\ \begin{tabular}{ll|l} 
and Biochemistry & $\begin{array}{l}\text { DOI: } 10.33594 / 000000043 \\
\text { Published online: } 26 \text { March } 2019\end{array}$ & $\begin{array}{l}\text { C } 2019 \text { The Author(s). Published by } \\
\text { Cell Physiol Biochem Press GmbH\&Co. KG }\end{array}$
\end{tabular} \\ Zhu et al.: Effect of miR-106b on Metastasis of Early Gastric Cancer}

\section{TUNEL staining}

Tissues were embedded and incubated with proteinase $\mathrm{K}(40 \mu \mathrm{g} / \mathrm{mL})$ for 1 hour at $37^{\circ} \mathrm{C}$, then treated with $2 \% \mathrm{H}_{2} \mathrm{O}_{2}$ in distilled water for $30 \mathrm{~min}$ at $37^{\circ} \mathrm{C}$. After enzymatic reaction, sections were washed with PBS, incubated with anti-digoxigenin peroxidase conjugate for $30 \mathrm{~min}$ at $37^{\circ} \mathrm{C}$ in a humified chamber. Sections were stained with diaminobenzine and counterstained with hematoxylin, and observed under a light microscope.

\section{Statistical analysis}

The data were expressed as the means \pm standard deviations of three independent experiments and data were considered statistically significant when values reached $\mathrm{P}<0.05$.

\section{Results}

Effect of miR-106b on the metastasis of EGC

The expression of miR-106b was explored in the tissues of patients with EGC metastasis by Q-PCR and FISH. The result of Q-PCR revealed that the expression of miR-106b in the metastasis tissues showed nearly 2-fold increase compared with non-metastasis tissues (Fig. 1a). Moreover, FISH result showed that the expression of miR-106b was slightly increased in the metastasis tissues compared with non-metastasis tissues (Fig. 1b). To investigate the effect of miR-106b on the metastasis of EGC, we up-regulated and down-regulated the expression of miR-106b by the mimics or inhibitor of miR-106b in gastric cancer cells. The result showed the expression of miR-106b was significantly decreased following treatment with the inhibitor of miR-106b (Fig. 1i), and significantly increased after treatment with miR106b mimics (Fig. 1c). The migration and invasion results revealed that miR-106b mimics treatment significantly increased the migrated (Fig. 1d and e) and invaded (Fig. 1f and g) cells when compared with the control. Meanwhile, miR-106b mimics also promoted the expression levels of EMT-related proteins, including SNAI2, ZEB1, TWIST1 and E-cadherin (Fig. 1h). On the other hand, we detected the effect of miR-106b inhibitor on the apoptosis of gastric cancer cells. The result showed that the apoptosis proportion was remarkably increased after treatment with miR-106b inhibitor compared with the control (Fig. 1j). In addition, miR-106b knockdown obviously enhanced the expression levels of apoptotic proteins such as Bax and Bim, and reduced Bcl-2 expression levels (Fig. 1k), which suggest miR-106b inhibitor could promote the apoptosis of gastric cancer cells.

\section{miR-106b targeted ALEX1 in gastric cancer cells}

ALEX1, a member of the armadillo family, is reported to inhibit cancer progress and metastasis. To study the effect of ALEX on miR-106b regulating the function of gastric cancer cells, we detected ALEX1 expression levels following treatment with or without miR-106b and up-regulated ALEX1 by infection of PLVX-ALEX1 (Fig. 2a and b). The result showed that miR-106b inhibitor obviously enhanced the expression levels of ALEX and miR-106b mimics reduced ALEX1 expression levels in AGS cells (Fig. 2a). Moreover, in order to determine the relationship between ALEX1 and miR-106b in gastric cancer cells, the luciferase reporter plasmids containing the wt or mutant 3'-UTR segments of ALEX were constructed. The result demonstrated that the luciferase activities were remarkably promoted by miR-106b inhibitor combined with wt reporter plasmid treatment, while they were no difference in the treatment of miR-106b inhibitor combined with mutant reporter plasmid compared with the control. On the other hand, miR-106b mimics combined with wt reporter plasmid treatment significantly decreased the luciferase activities compared with the control. However, miR$106 \mathrm{~b}$ mimics combined with mutant reporter plasmid treatment showed no change of the luciferase activities (Fig. 2c). Interestingly, we also found the apoptotic cells induced by miR106b inhibitor were significantly decreased after cells were infected with PLVX-ALEX1 (Fig. $2 \mathrm{~d})$. In accordance with the result of apoptosis, the expression levels of apoptotic proteins were obviously reduced after combined treatment with miR-106b inhibitor and PLVX-ALEX1 


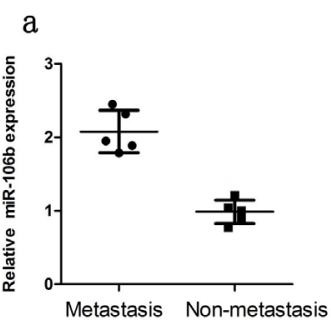

d

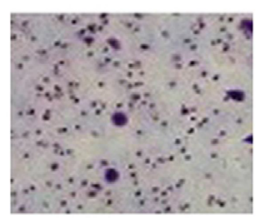

f

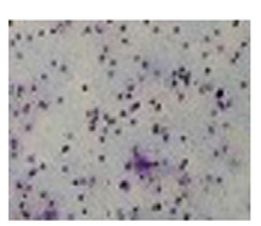

Control

$\mathrm{h}$

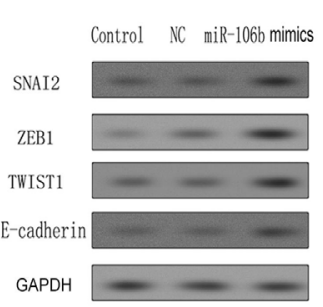

i b

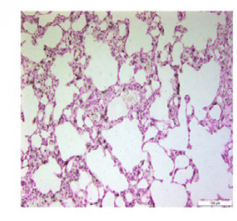

Metastasis

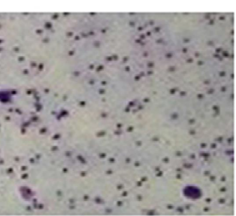

NC

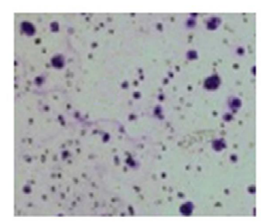

$\mathrm{NC}$

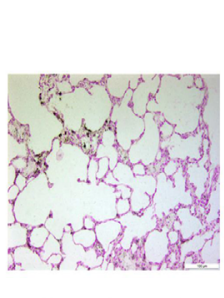

Non-metastasis

C

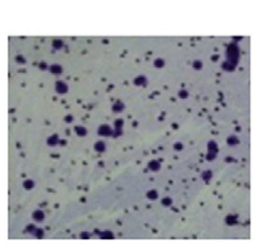

miR-106b mimcs

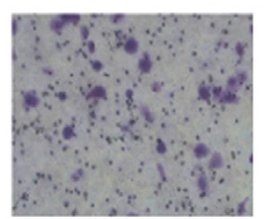

miR-106b mimcs
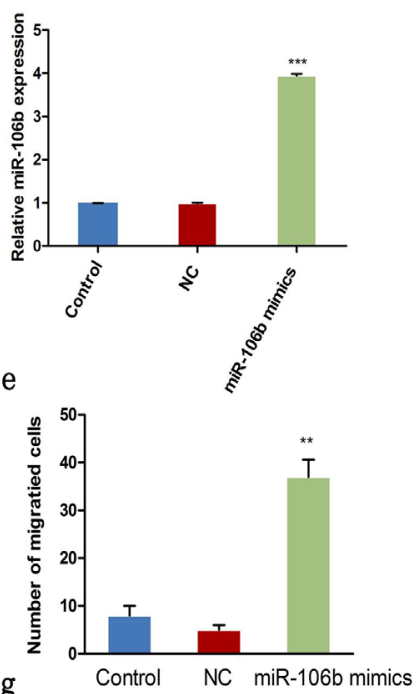

$\mathrm{g}$

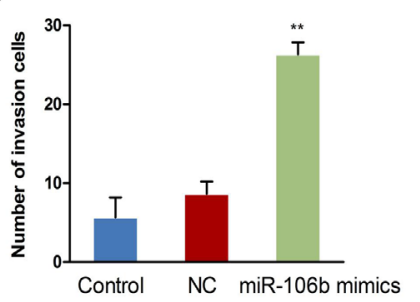

j

K
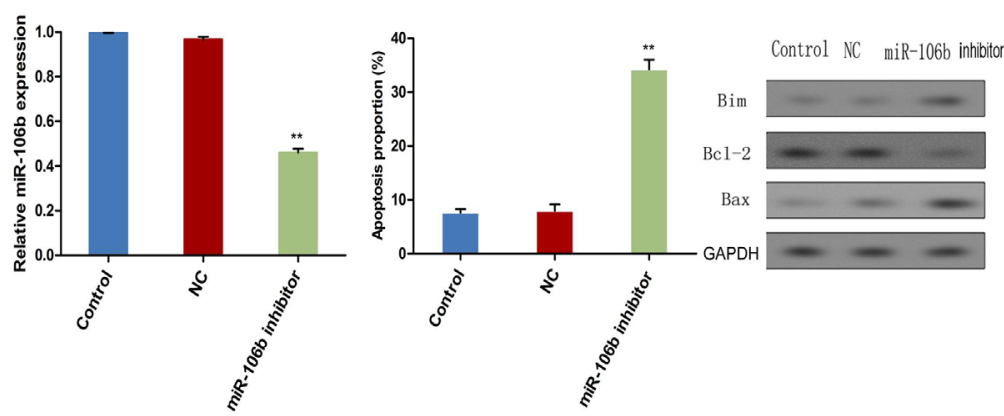

Fig. 1. Effect of miR-106b on migration and invasion of gastric cancer cells. (a, b) miR-106b expression was detected in the tissues of EGC by Q-PCR and FISH. (c) Expression of miR-106b was verified following treatment with miR-106b mimics. (d, e) Migration ability of gastric cancer cells was detected following treatment with miR-106b mimics. (f, g) Invasion ability of gastric cancer cells was detected following treatment with miR-106b mimics. (h) Detection of EMT related proteins SNAI2, ZEB1, TWIST1, E-cadherin after cells treated with miR-106b inhibitor. (i) Expression of miR-106b was verified following treatment with miR-106b inhibitor. (j) AGS cells were treated with miR-106b inhibitor, then stained with annexin V-FITC/7-AAD and analyzed by flow cytometry. (k) Apoptotic protein expression levels of Bim, Bcl-2, Bax were detected following treatment with miR-106b inhibitor. ${ }^{* *} \mathrm{P}<0.01$ versus control. ${ }^{* * *} \mathrm{P}<0.001$ versus control.

when compared with miR-106b inhibitor treatment alone (Fig. $2 \mathrm{~g}$ and $2 \mathrm{~h}$ ). In addition, the EMT related protein expression levels were decreased following combined treatment with miR-106b inhibitor and PLVX-ALEX1 when compared with miR-106b mimics treatment alone (Fig. 2e and 2f ). 


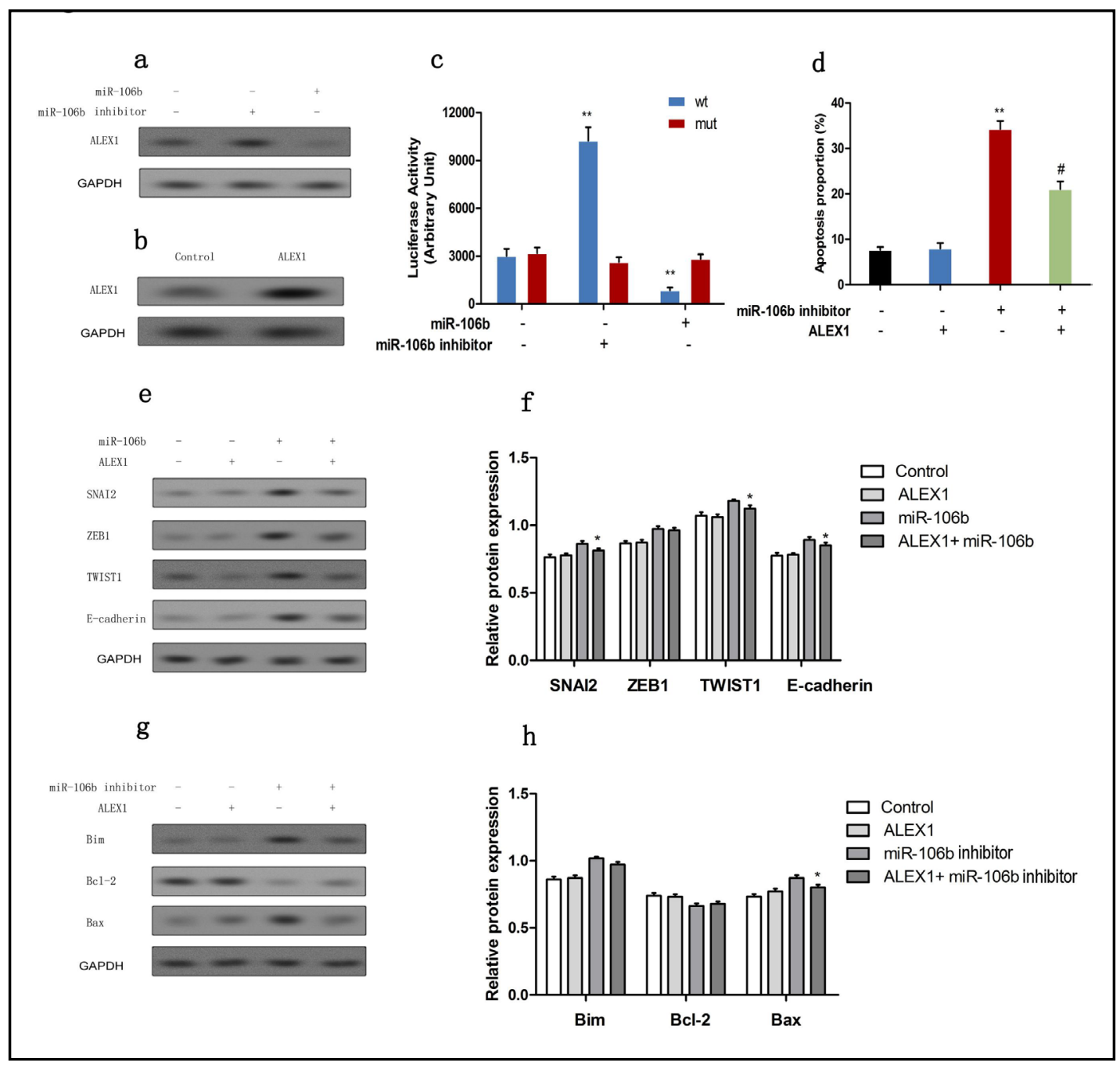

Fig. 2. miR-106b targeted ALEX1 in gastric cancer cells. (a) miR-106b mimics and miR-106b inhibitor were used to treat gastric cancer cells, and the expression of ALEX1 was detected. (b) ALEX1 expression level was detected following PLVX-ALEX1 treatment. (c) Luciferase activity of ALEX1 was detected by luciferase reporter assay following treatment with miR-106b mimics or miR-106b inhibitor. ${ }^{* * P<0.01}$. (d) Cell apoptosis was detected following treatment with miR-106b inhibitor and/or PLVX-ALEX1. ${ }^{* *} \mathrm{P}<0.01$ versus control. \# $\mathrm{P}<0.05$ versus miR-106b inhibitor treatment. (e, f) Detection of EMT related proteins SNAI2, ZEB1, TWIST1, E-cadherin after cells treated with miR-106b mimics and/or PLVX-ALEX1. *P<0.05 versus miR-106b mimics treatment. (g, h) Detection of apoptotic proteins after cells treated with miR-106b inhibitor and/or PLVX-ALEX1. ${ }^{*} \mathrm{P}<0.05$ versus miR-106b inhibitor treatment.

Down-regulation of miR-106b promoted apoptosis of gastric cancer cells through JAK1/ STAT3 signaling pathway

To further investigate the molecular mechanism of miR-106b mediated apoptosis of gastric cancer cells, JAK/STAT signaling pathway related proteins were studied after treatment with miR-106b inhibitor. The results revealed that miR-106b inhibitor treatment led to a decrease of pJAK1 and pSTAT3 expression levels compared with the control (Fig. 3a). In order to study the effect of ALEX1 on miR-106b inhibitor regulating JAK/STAT signaling pathway, the gastric cancer cells were infected with PLVX-ALEX1 following treatment with miR-106b inhibitor. As expected, PLVX-ALEX1 infection treatment reversed the expression levels of pJAK1 and pSTAT3 inhibited by miR-106b inhibitor (Fig. 3b). The relative expression levels of pJAK1 and pSTAT3 were significantly increased by PLVX-ALEX1 combined with miR- 


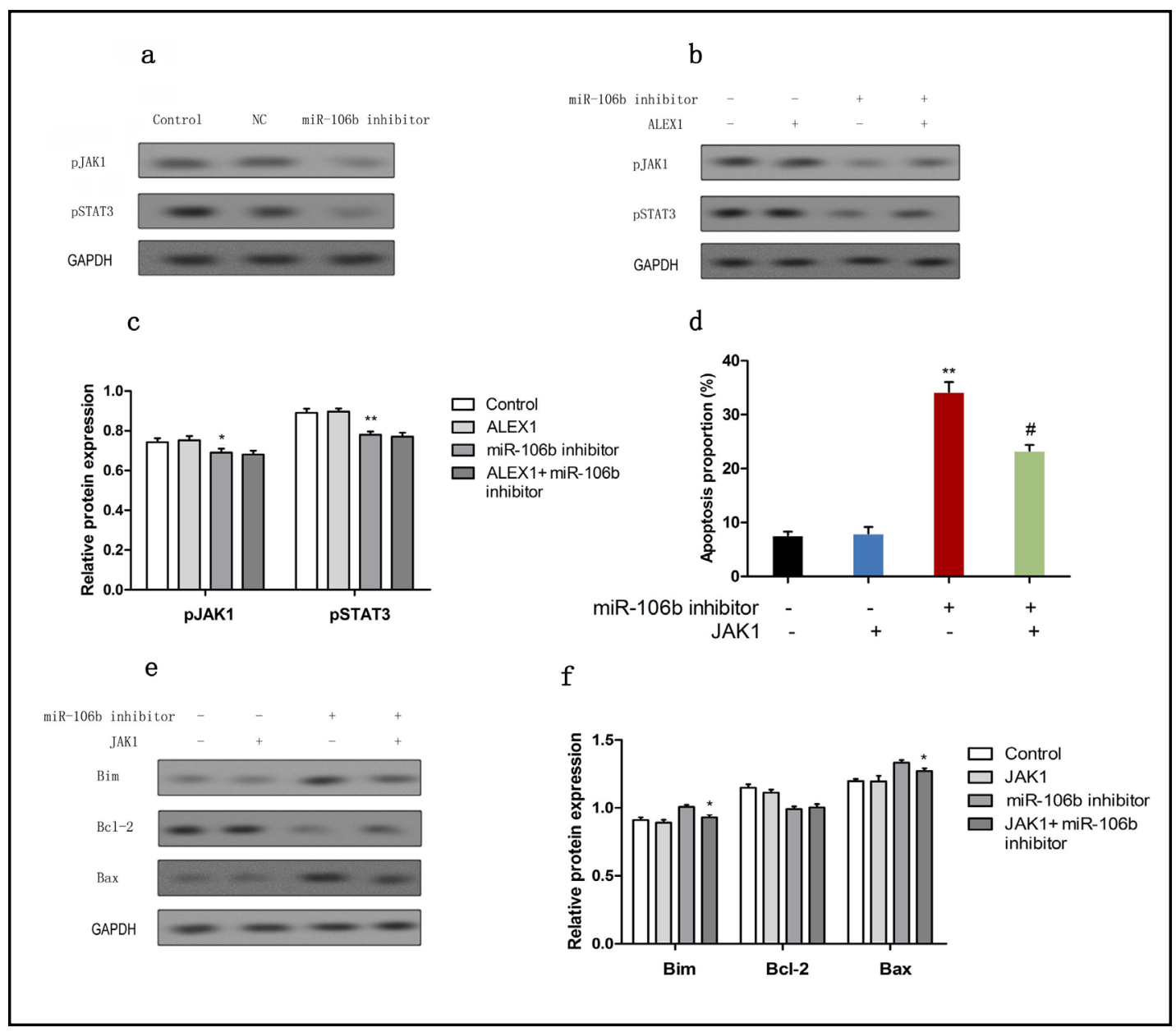

Fig. 3. miR-106b inhibitor regulated JAK1/STAT3 Signaling Pathway. (a) Detection of JAK/STAT pathway related protein levels after cells treated with or without miR-106b inhibitor. (b, c) The expression levels of pJAK1 and pSTAT3 were detected following treatment with with miR-106b inhibitor and/or PLVX-ALEX1. ${ }^{*} \mathrm{P}<0.05$ versus control, ${ }^{* *} \mathrm{P}<0.01$ versus control. (d) Cell apoptosis was detected following treatment with miR-106b inhibitor and/or PLVX-JAK1. ${ }^{*} \mathrm{P}<0.01$ versus control. \# $\mathrm{P}<0.05$ versus miR-106b inhibitor treatment. (e, f) Detection of apoptotic proteins after cells treated with miR-106b inhibitor and/or PLVXJAK1. ${ }^{*} \mathrm{P}<0.05$ versus miR-106b inhibitor treatment.

106b inhibitor treatment when compared with miR-106b inhibitor treatment alone (Fig. 3c). To further determine the effect of JAK/STAT signaling pathway on miR-106b inhibitor induced apoptosis, we constructed the PLVX-JAK1 vectors and infected the gastric cancer cells. The result showed that PLVX-JAK1 combined with miR-106b inhibitor treatment remarkably decreased the apoptotic cells when compared with miR-106b inhibitor treatment alone (Fig. $3 \mathrm{~d}$ ). Consistently, the apoptotic protein expression levels of Bax and Bim were obviously reduced, and the Bcl-2 expression levels were increased after PLVX-ALEX1 combined with miR-106b inhibitor treatment (Fig. 3e and f).

\section{Down-regulation of miR-106b inhibited gastric cancer in vivo}

To testify the effect of miR-106b knockdown on gastric cancer in vivo, we constructed the mouse model of gastric cancer using AGS gastric cancer cells. The tumor volume was observed in different treatments for indicated time. The result revealed miR-106b inhibitor significantly reduced the tumor volume of gastric cancer, and this effect was further promoted by the treatment of GLPG0643 (Fig. 4a). Moreover, down-regulation of miR-106b enhanced the protein expression levels of ALEX1 in vivo (Fig. 4b). Meanwhile, down-regulation of miR- 


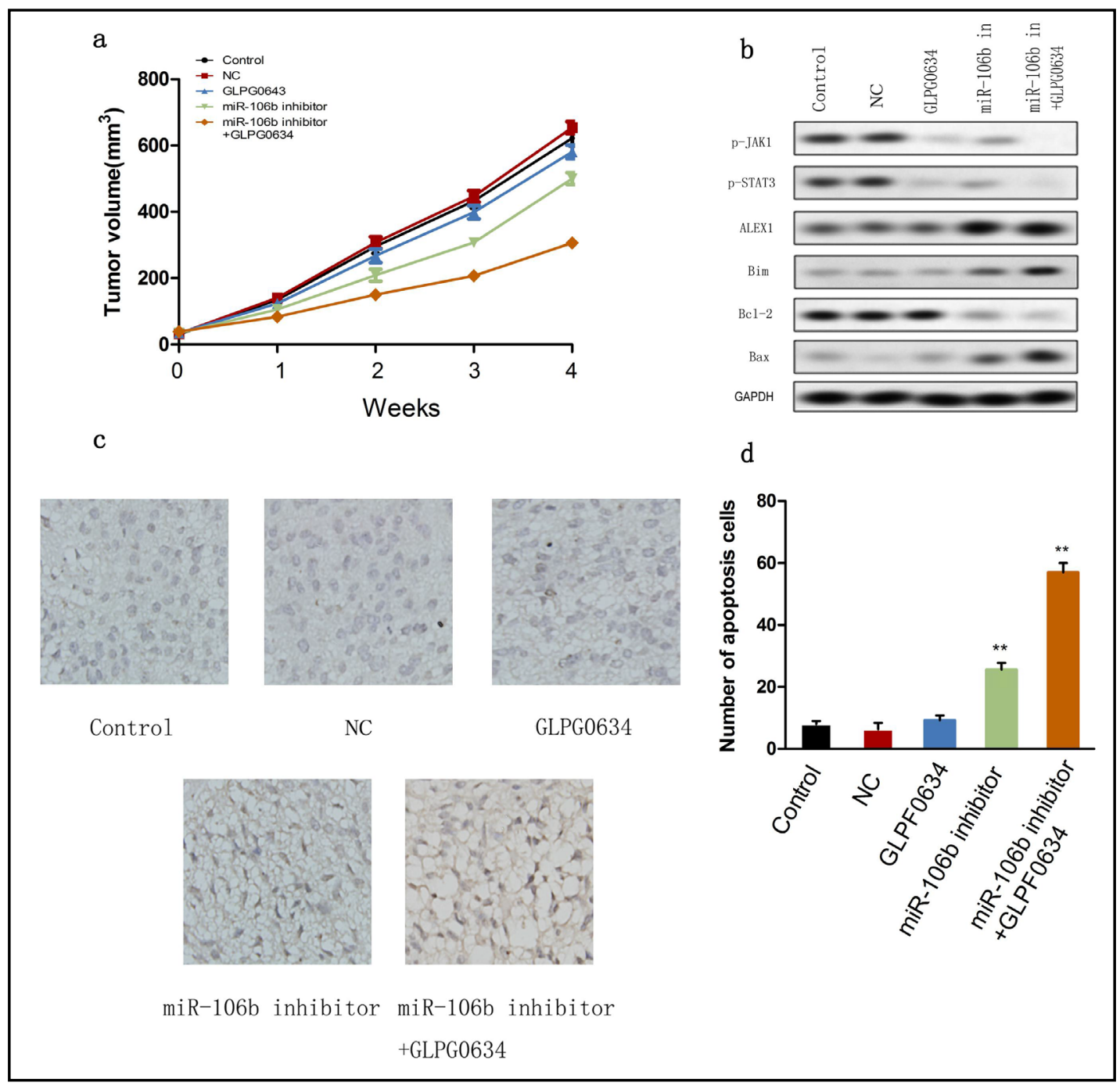

Fig. 4. miR-106b inhibited gastric cancer in vivo. (a) Tumor volume was measured with different treatments for indicated time points. (b) The protein expression levels of pJAK1, pSTAT3, ALEX1, Bim, Bcl-2 and Bax were detected following with different treatments. (c, d) Apoptosis was detected using TUNEL staining for the tumor tissue treated with different treatments. ${ }^{* *} \mathrm{P}<0.01$ VS Control.

106b significantly decreased the pJAK1 and pSTAT3 protein expression levels compared with the control, which was consistent with the results in vitro, and the effect of miR-106b knockdown on the pJAK1 and pSTAT3 protein expression was enhanced by the treatment of GLPG0643 (Fig. 4b). To further determine the role of miR-106b knockdown in gastric cancer, the apoptosis of tumor tissues was detected using a TUNEL assay. The result revealed that the TUNEL positive cells were remarkably increased by down-regulation of miR-106b when compared with the control, and further promoted by GLPG0643 treatment (Fig. 4c and d). In addition, the apoptotic protein expression levels of Bax and Bim were obviously increased, and the Bcl-2 expression levels were decreased following down-regulation of miR-106b, which further strengthened by GLPG0643 treatment (Fig. 4b). 


\section{Cellular Physiology Cell Physiol Biochem 2019;52:606-616 \\ \begin{tabular}{ll|l} 
and Biochemistry $\begin{array}{l}\text { DOl: 10.33594/000000043 } \\
\text { Published online: 26 March 2019 }\end{array}$ & $\begin{array}{l}\text { O } 2019 \text { The Author(s). Published by } \\
\text { Cell Physiol Biochem Press GmbH\&Co. KG }\end{array}$ \\
\cline { 2 - 3 } &
\end{tabular} \\ Zhu et al.: Effect of miR-106b on Metastasis of Early Gastric Cancer}

\section{Discussion}

miR-106b is reported to overexpression in various types of cancers and plays an important role in the regulation of cell invasion and migration in vitro and in vivo [26-29]. However, the expression of miR-106b and its possible functions in metastasis of cancer cells are not clear in EGC. In this study, the expression levels of miR-106b in metastasis tissues of EGC were increased when compared with non-metastasis tissues. ALEX1, a novel tumor suppressor gene, suppresses metastasis of gastric cancer, which involved in PAR-1/Rho GTPase signaling pathway [30]. The tentative inhibition of miR-106b in metastasis of EGC and the possible mechanism are urgent to be solved.

Previous study demonstrated that miR-106b was up-regulated in cancer associated fibroblasts (CAFs) from patients with gastric cancer, and CAFs with down-regulated miR106b could significantly inhibit gastric cancer cell migration and invasion by targeting PTEN [31]. In this study, we revealed the up-regulation of miR-106b in the metastasis tissues from patients with EGC. In vitro results showed that the migration and invasion ability of gastric cancer cells were significantly increased following treatment with miR-106b mimics. On the other hand, compared with the control, miR-106b inhibitor treatment showed a high level of apoptosis proportion, as well as the expression of apoptotic proteins in gastric cancer cells. Furthermore, the expression levels of ALEX were promoted by the treatment of miR-106b inhibitor, and reduced by the treatment of miR-106b mimics. The luciferase reporter result demonstrated that ALEX1 was a direct target of miR-106b in gastric cancer cells. Overexpression of ALEX1 rescued the cell apoptosis and apoptotic proteins expression levels induced by miR-106b inhibitor, which was in accordance with the result of previous study [25]. Moreover, we noted that phosphorylation of JAK1 and phosphorylation of STAT3 were suppressed by miR-106b inhibitor treatment, which partially reversed following upregulation of ALEX1. JAK1/STAT3 signaling pathway was reported to serve as a target for gastric cancer treatment [32]. A recent study showed that inhibiting STAT3 phosphorylation involved in the suppression of inflammation and proliferation in gastric cancer cells [33]. Interestingly, we found overexpression of JAK1 reduced apoptosis and apoptotic proteins expression levels in gastric cancer cells. Moreover, GLPG0643 treatment enhanced the inhibitory effect of miR-106b inhibitor on gastric cancer growth in vivo. The TUNEL positive cells were also significantly increased by the combined treatment of GLPG0643 and miR-106b inhibitor when compared with the control. In addition, the apoptotic protein expression levels of Bax, Bim were further strengthened by GLPG0643 combined with miR$106 \mathrm{~b}$ inhibitor treatment. These results suggested that JAK1 might be a tentative target that enhanced the effect of miR-106b inhibitor on the suppression of gastric cancer growth.

\section{Conclusion}

Taken together, miR-106b was a potent factor of promoting metastasis of EGC. Moreover, miR-106b inhibitor exhibited anti-cancer characteristics both in vitro and in vivo. The identification of ALEX1 expression correlating to miR-106b highlighted the significance of ALEX1 in miR-106b inhibitor suppressed gastric cancer, suggesting ALEX1 was a direct target of miR-106b. In addition, down-regulation of miR-106b promoted apoptosis of gastric cancer cells through inhibiting JAK/STAT signaling pathway. These findings could be translational into clinical trials and would shed light on the potential and valid strategy in the treatment of EGC. 


\section{Cellular Physiology Cell Physiol Biochem 2019;52:606-616 \begin{tabular}{l|l|l|l} 
DOI: 10.33594/000000043 2019 The Author(s). Published by \\
\hline
\end{tabular} \\ Published online: 26 March 2019 Cell Physiol Biochem Press GmbH\&Co. KG \\ Zhu et al.: Effect of miR-106b on Metastasis of Early Gastric Cancer}

\section{Acknowledgements}

This work was supported by the National Science Foundation of China (81672822).

\section{Disclosure Statement}

The authors declare that they have no competing interests.

\section{References}

1 Harada K, Mizrak Kaya D, Shimodaira Y, Ajani JA: Global chemotherapy development for gastric cancer. Gastric Cancer 2017;20:92-101.

2 Sano T: Gastric cancer: Asia and the world. Gastric Cancer 2017;20:1-2.

- 3 Zong L, Abe M, Seto Y, Ji J: The challenge of screening for early gastric cancer in China. Lancet 2016;388:2606.

4 Kim KH, Kim CG, Kim YW, Moon H, Choi JE, Cho SJ, Lee JY, Choi IJ: Phlegmonous Gastritis with Early Gastric Cancer. J Gastric Cancer 2016;16:195-199.

5 Sekikawa A, Fukui H, Maruo T, Tsumura T, Okabe Y, Osaki Y: Diabetes mellitus increases the risk of early gastric cancer development. Eur J Cancer 2014;50:2065-2071.

6 Shin N, Jeon TY, Kim GH, Park DY: Unveiling lymph node metastasis in early gastric cancer. World J Gastroenterol 2014;20:5389-5395.

7 Yoon H, Kim N: Diagnosis and management of high risk group for gastric cancer. Gut Liver 2015;9:5-17.

- 8 Jeon MY, Park JC, Hahn KY, Shin SK, Lee SK, Lee YC: Long-term outcomes after noncurative endoscopic resection of early gastric cancer: the optimal time for additional endoscopic treatment. Gastrointest Endosc 2018;87:1003-1013.e2.

9 Kim JY, Kim CH, Lee Y, Lee JH, Chae YS: Tumour infiltrating lymphocytes are predictors of lymph node metastasis in early gastric cancers. Pathology 2017;49:589-595.

10 Fang WL, Huang KH, Lan YT, Chen MH, Chao Y, Lo SS, Wu CW, Shyr YM, Li AF: The Risk Factors of Lymph Node Metastasis in Early Gastric Cancer. Pathol Oncol Res 2015;21:941-946.

- 11 Barreto SG, Windsor JA: Redefining early gastric cancer. Surg Endosc 2016;30:24-37.

- 12 Jo MJ, Park JY, Song JS, Kook MC, Ryu KW, Cho SJ, Lee JH, Nam BH, Hong EK, Choi IJ, Kim YW: Biopathologic features and clinical significance of micrometatasis in the lymph node of early gastric cancer. World J Gastroenterol 2015;21:667-674.

- 13 Lee YS, Dutta A: MicroRNAs in cancer. Annu Rev Pathol 2009;4:199-227.

- 14 Bueno MJ, Perez de Castro I, Malumbres M: Control of cell proliferation pathways by microRNAs. Cell Cycle 2008;7:3143-3148.

- 15 Babashah S, Soleimani M: The oncogenic and tumour suppressive roles of microRNAs in cancer and apoptosis. Eur J Cancer 2011;47:1127-1137.

16 Li Z, Lei H, Luo M, Wang Y, Dong L, Ma Y, Liu C, Song W, Wang F, Zhang J, Shen J, Yu J: DNA methylation downregulated miR-10b acts as a tumor suppressor in gastric cancer. Gastric Cancer 2015;18:43-54.

17 Zhao Y, Lu G, Ke X, Lu X, Wang X, Li H, Ren M, He S: miR-488 acts as a tumor suppressor gene in gastric cancer. Tumour Biol 2016;37:8691-8698.

18 Wang Y, Liu C, Luo M, Zhang Z, Gong J, Li J, You L, Dong L, Su R, Lin H, Ma Y, Wang F, Wang Y, Chen J, Zhang J, Jia H, Kong Y, Yu J: Chemotherapy-Induced miRNA-29c/Catenin-delta Signaling Suppresses Metastasis in Gastric Cancer. Cancer Res 2015;75:1332-1344.

19 Li N, Miao Y, Shan Y, Liu B, Li Y, Zhao L, Jia L: miR-106b and miR-93 regulate cell progression by suppression of PTEN via PI3K/Akt pathway in breast cancer. Cell Death Dis 2017;8:e2796.

20 Yau WL, Lam CS, Ng L, Chow AK, Chan ST, Chan JY, Wo JY, Ng KT, Man K, Poon RT, Pang RW: Over-expression of miR-106b promotes cell migration and metastasis in hepatocellular carcinoma by activating epithelialmesenchymal transition process. PloS One 2013;8:e57882. 


\section{Cellular Physiology Cell Physiol Biochem 2019;52:606-616

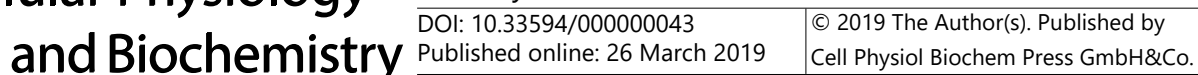 \\ Zhu et al.: Effect of miR-106b on Metastasis of Early Gastric Cancer}

21 Zeng Q, Jin C, Chen W, Xia F, Wang Q, Fan F, Du J, Guo Y, Lin C, Yang K, Li J, Peng X, Li X, Cao K:

Downregulation of serum miR-17 and miR-106b levels in gastric cancer and benign gastric diseases. Chin J Cancer Res 2014;26:711-716.

- 22 Zheng Z, Zhang Y, Zhang Z, Yang Y, Song T: Effect of miR-106b on Invasiveness of Pituitary Adenoma via PTEN-PI3K/AKT. Med Sci Monitor 2017;23:1277-1285.

- 23 Dai F, Liu T, Zheng S, Liu Q, Yang C, Zhou J, Chen Y, Sheyhidin I, Lu X: miR-106b promotes migration and invasion through enhancing EMT via downregulation of Smad 7 in Kazakh's esophageal squamous cell carcinoma. Tumour Biol 2016;37:14595-14604.

- 24 Iseki H, Takeda A, Andoh T, Kuwabara K, Takahashi N, Kurochkin IV, Ishida H, Okazaki Y, Koyama I: ALEX1 suppresses colony formation ability of human colorectal carcinoma cell lines. Cancer Sci 2012;103:12671271.

25 Gao Y, Wu JY, Zeng F, Liu GL, Zhang HT, Yun H, Song FZ: ALEX1 Regulates Proliferation and Apoptosis in Breast Cancer Cells. Asian Pac J Cancer 2015;16:3293-3299.

26 Ni X, Xia T, Zhao Y, Zhou W, Wu N, Liu X, Ding Q, Zha X, Sha J, Wang S: Downregulation of miR-106b induced breast cancer cell invasion and motility in association with overexpression of matrix metalloproteinase 2. Cancer Sci 2014;105:18-25.

- 27 Wang YX, Lang F, Liu YX, Yang CQ Gao HJ: In situ hybridization analysis of the expression of miR-106b in colonic cancer. Int J Clin Exp Patho 2015;8:786-792.

- 28 Piao J, You K, Guo Y, Zhang Y, Li Z, Geng L: Substrate stiffness affects epithelial-mesenchymal transition of cervical cancer cells through miR-106b and its target protein DAB2. Int J Oncol 2017;50:2033-2042.

- 29 Wei K, Pan C, Yao G, Liu B, Ma T, Xia Y, Jiang W, Chen L, Chen Y: miR-106b-5p Promotes Proliferation and Inhibits Apoptosis by Regulating BTG3 in Non-Small Cell Lung Cancer. Cell Physiol Bioche 2017;44:15451558.

- 30 Pang L, Li JF, Su L, Zang M, Fan Z, Yu B, Wu X, Li C, Yan M, Zhu ZG, Liu B: ALEX1, a novel tumor suppressor gene, inhibits gastric cancer metastasis via the PAR-1/Rho GTPase signaling pathway. J Gastroenterol 2018;53:71-83.

31 Yang TS, Yang XH, Chen X, Wang XD, Hua J, Zhou DL, Zhou B, Song ZS: microRNA-106b in cancer-associated fibroblasts from gastric cancer promotes cell migration and invasion by targeting PTEN. FEBS Lett 2014;588:2162-2169.

32 Su C, Wang W, Wang C: IGF-1-induced MMP-11 expression promotes the proliferation and invasion of gastric cancer cells through the JAK1/STAT3 signaling pathway. Oncol Lett 2018;15:7000-7006.

- 33 Song B, Zhan H, Bian Q Gu J: Piperlongumine inhibits gastric cancer cells via suppression of the JAK1, 2/ STAT3 signaling pathway. Mol Med Rep 2016;13:4475-4480. 